\title{
Chordoid Glioma
}

Singapore Med J 2015; 56(11): 641-643 doi: 10.11622/smedj.2015175

Dear Sir,

Chordoid glioma is a very rare, slow-growing tumour of the central nervous system (CNS) which arises from the anterior portion of the third ventricle, as first described by Brat et $\mathrm{al}^{(1)}$ in 1998. It was included in the classification of tumours of the CNS by the World Health Organization in 2000. ${ }^{(2)}$ This tumour usually presents in adults in the reported age range of 5-71 years, ${ }^{(3)}$ with a female predilection of $3: 1{ }^{(2)}$ However, three cases have been reported in children aged between five and 12 years, ${ }^{(3)}$ and in children, the tumour may not be confined to the third ventricle. Common clinical features at presentation are persistent headaches, visual disturbances with or without a field defect, memory disturbances and endocrine abnormalities. ${ }^{(2)}$

Our patient is a 30-year-old Chinese woman who presented to a private ophthalmologist on 19 September 2014 because of recent problems with her peripheral vision. Her visual field examination showed a bitemporal hemianopia (Fig. 1) which was denser in the right temporal field, while the rest of the ocular examination, including that of her optic nerves, was normal. Magnetic resonance (MR) imaging showed an isointense lesion with solid heterogeneous postgadolinium enhancement on T1-weighted imaging and hyperintensity on T2. There was peritumoural oedema and a compressive effect upon the optic chiasm from above, distorting it inferiorly, although it was not enhanced (Figs. 2a-c). The pituitary stalk was displaced posteriorly, but the positioning of the pituitary gland was preserved (Fig. 2d). The differential diagnosis suggested was either a germinoma or, more likely, a chordoid glioma.

The patient was referred to our neurosurgeon and underwent right subfrontal craniotomy on 26 September 2014. The tumour, which was adherent to the floor of the third ventricle and right side of the hypothalamus, was carefully dissected from the adjacent structures. Gross total resection was achieved over a five-hour procedure. The intraoperative histopathology from a frozen section showed a cellular tumour consisting of cords and nests of eosinophilic epithelioid cells in a vacuolated background of basophilic myxoid material. Focal aggregates of lymphoplasmacytic cells were also seen. As the tumour cells were positive for glial fibrillary acidic protein (GFAP) uptake, the diagnosis of chordoid glioma was proposed, which was later confirmed in the final histopathology report. The patient's postoperative course was uneventful apart from the development of transient diabetes insipidus and hyponatraemia in the immediate postoperative period. On her follow-up visit to our neuro-ophthalmology clinic one week after surgery, her visual fields were found to be normal (Fig. 3) and remained so four months later. MR imaging eight weeks after the operation showed no evidence of a residual tumour (Fig. 4).
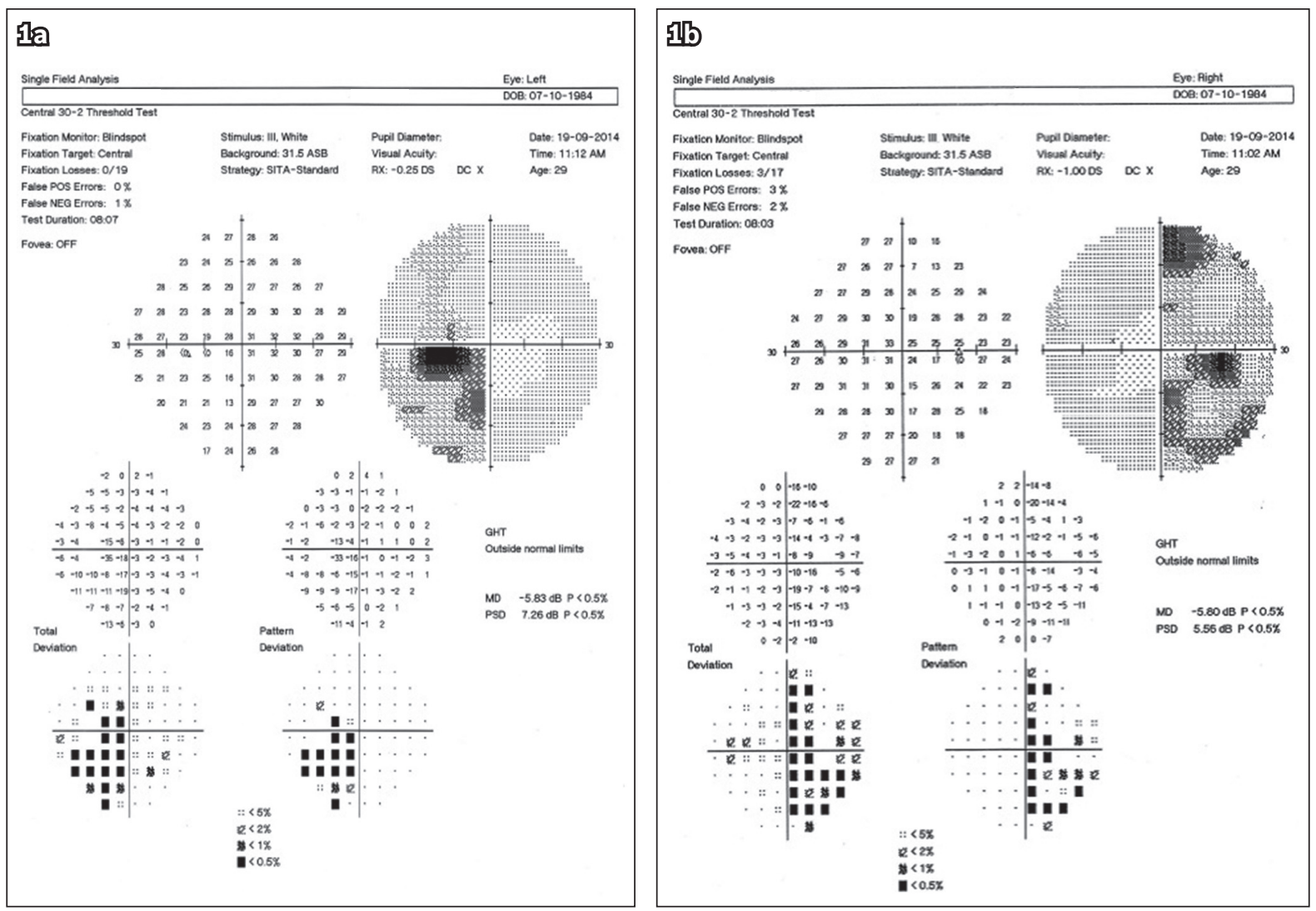

Fig. 1 ( $a$ \& b) Preoperative Humphrey visual field analysis shows a bitemporal hemianopia which is denser in the right temporal field. 

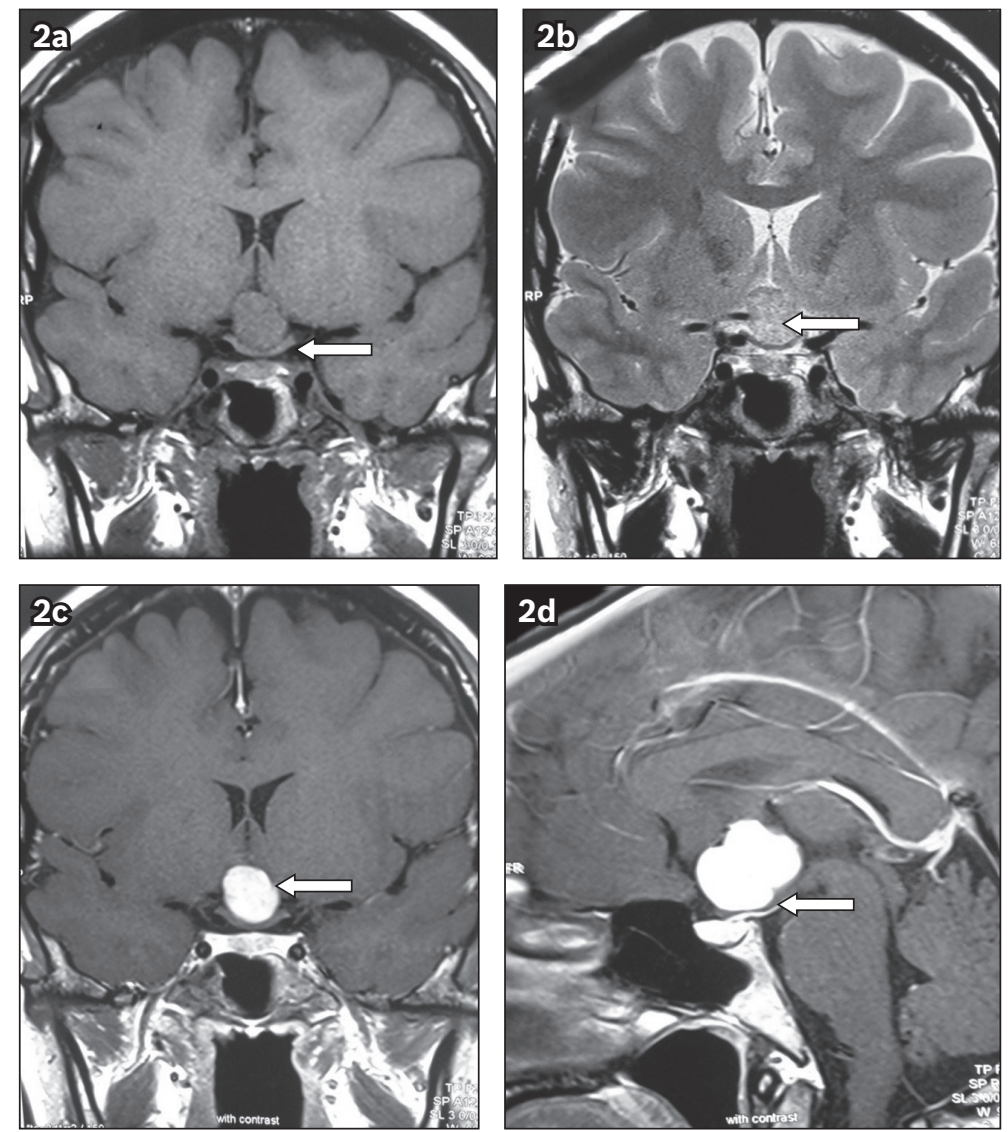

Fig. 2 Preoperative MR images show (a) T1-W coronal view with an isointense suprasellar lesion causing inferior bowing of the optic chiasm (arrow); (b) T2-W coronal view with a hyperintense suprasellar lesion (arrow); (c) T1-W coronal view postgadolinium with heterogeneous enhancement of the suprasellar lesion (arrow); and (d) T1-W sagittal view postgadolinium enhancement with a distorted pituitary stalk (arrow) and normal pituitary gland positioning.
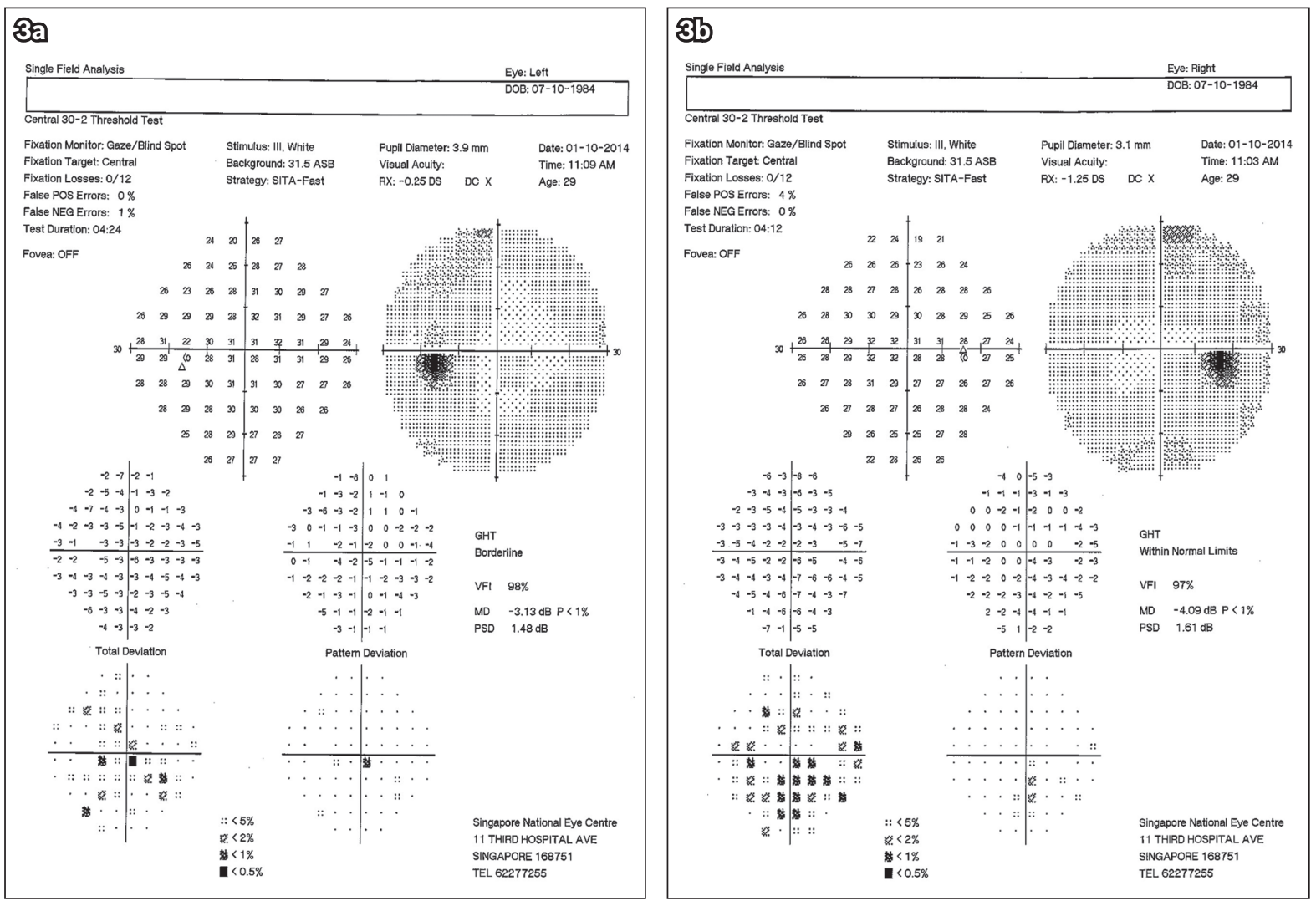

Fig. 3 (a \& b) Postoperative Humphrey visual field analysis at three weeks shows resolution of the bitemporal defects. 


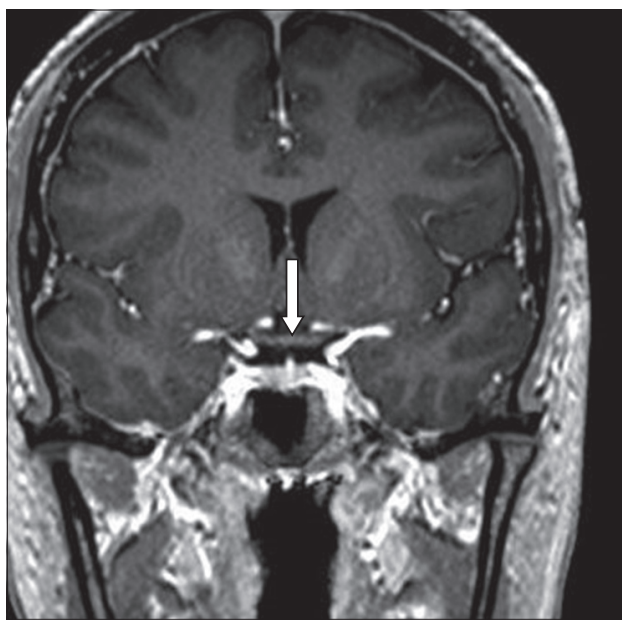

Fig. 4 T1-W MR image shows postgadolinium corona view eight weeks after surgery with no evidence of tumour and a normal optic chiasm (arrow).

Chordoid glioma is a very rare tumour, having both chordoid and glial characteristics, and is most commonly found in the area of the third ventricle adjacent to the hypothalamus, optic chiasm and optic nerves. ${ }^{(3)}$ It has rarely been described in other areas of the brain such as the temporoparietal cortex or thalamus, such locations being more likely to be seen in children. ${ }^{(2)}$ Because of the proximity of these tumours to the optic chiasm, visual field defects and optic neuropathy have been described as presenting features, mimicking those associated with pituitary tumours. Our patient presented with a bitemporal hemianopia due to the tumour's impingement on the chiasm. Al Hinai et al, ${ }^{(4)}$ in their review of 65 reported cases of chordoid gliomas, stated that none of these cases were shown to have actual invasion of the optic chiasm. However, their own further case report ${ }^{(4)}$ was of a 50 -year-old woman who displayed an enlarged optic chiasm and postgadolinium enhancement on MR imaging, and who was found at operation to have actual tumour invasion of the chiasm.

The typical radiological features of chordoid glioma of the third ventricle are isointensity on T1-weighted imaging and isointensity or hyperintensity on T2, with postgadolinium homogeneous or heterogeneous enhancement. ${ }^{(2,3)}$ Areas of vasogenic oedema may also be present, causing compression of the surrounding structures, particularly the visual pathway. A cystic or lobulated component may be seen. ${ }^{(2)}$ Similar MR imaging findings in our patient were reported, and at operation, we found a firm, yellowish tumour adherent to the third ventricle and extending into the suprasellar region, compressing the optic chiasm and bowing it inferiorly. The histological characteristics of a chordoid glioma are cords and clusters of epithelioid cells with a mucinous background and low-grade lymphocytic infiltrates. These elements are of glial origin and such features are not found in germinomas. ${ }^{(5)}$ Taken together, the presence of glial elements and the uptake of GFAP favour the diagnosis of chordoid glioma. Brat et al, ${ }^{(1)}$ who were the first to describe these features, subsequently proposed that chordoid glioma should be classified as a separate pathological entity.

Yours sincerely,

Lakana Kumar $\underline{\text { Thavaratnam }}{ }^{1}$, Shun Ting $\underline{\text { Loy }}^{2}$, Arvind $\underline{\text { Gupta }}^{1}$, Ivan $\underline{\mathrm{Ng}}^{2}$, James F $\underline{\text { Cullen }}^{1}$

${ }^{1}$ Neuro-Ophthalmology Department, Singapore National Eye Centre, 2Neurosurgery Service, Singapore General Hospital, Singapore. jbarrycullen@yahoo.com

\section{References}

1. Brat DJ, Scheithauer BW, Staugaitis SM, et al. Third ventricular chordoid glioma: a distinct clinicopathologic entity. J Neuropathol Exp Neurol 1998; 57:283-90.

2. Desouza RM, Bodi I, Thomas N, Marsh H, Crocker M. Chordoid glioma: ten years of a low-grade tumor with high morbidity. Skull Base 2010; 20:125-38.

3. Kobayashi T, Tsugawa T, Hashizume C, et al. Therapeutic approach to chordoid glioma of the third ventricle. Neurol Med Chir (Tokyo) 2013; 53:249-55.

4. Al Hinai QS, Petrecca K. Rarest of the rare: Chordoid glioma infiltrating the optic chiasm. Surg Neurol Int 2011; 2:53.

5. Pomper MG, Passe TJ, Burger PC, Scheithauer BW, Brat DJ. Chordoid glioma: a neoplasm unique to the hypothalamus and anterior third ventricle. AJNR Am J Neuroradiol 2001; 22:464-9. 